DAMTP-2004-113

hep-th/0410185

\title{
Spacetime-Filling Branes in Ten and Nine Dimensions
}

\author{
Fabio Riccioni \\ DAMTP \\ Centre for Mathematical Sciences \\ University of Cambridge \\ Wilberforce Road Cambridge CB3 OWA \\ $U K$
}

\begin{abstract}
Type-IIB supergravity in ten dimensions admits two consistent $Z_{2}$ truncations. After the insertion of D9-branes, one of them leads to the low-energy action of type-I string theory, and it can be performed in two different ways, in correspondence with the fact that there are two different consistent ten-dimensional type-I string theories, namely the $S O(32)$ superstring and the $U S p(32)$ model, in which supersymmetry is broken on the D9-branes. We derive here the same results for Type-IIA theory compactified on a circle in the presence of D8-branes. We also analyze the $\kappa$-symmetric action for a brane charged with respect to the S-dual of the RR 10-form of type-IIB, and we find that the tension of such an object has to scale like $g_{S}^{-2}$ in the string frame. We give an argument to explain why this result is in disagreement with the one obtained using Weyl rescaling of the brane action, and we argue that this brane can only be consistently introduced if the other $Z_{2}$ truncation of type-IIB is performed. Moreover, we find that one can include a 10-form in type-IIA supersymmetry algebra, and also in this case the corresponding $\kappa$-symmetric brane has a tension scaling like $g_{S}^{-2}$ in the string frame.
\end{abstract}




\section{Introduction}

Type-II string theories in the non-perturbative regime contain in their spectrum BPS Dbranes, that are charged states with respect to RR fields [1], and are defined as hypersurfaces on which open strings end [2]. In the low-energy effective action, these states appear as $1 / 2$-supersymmetric solitonic solutions carrying electric or magnetic charge with respect to the RR fields of type-IIA and type-IIB supergravities. The effective action describing the massless modes of a D-brane is characterized by a Dirac-Born-Infeld (DBI) term and a Wess-Zumino (WZ) term. In $[3,4]$ it was shown that the effective action describing the massless open string states at string tree level is the DBI action in the approximation in which one neglects derivatives of the field strength, while the coupling to the RR fields is contained in the WZ term. The relative coefficient of the DBI and WZ terms is fixed, since the tension and the RR-charge of the brane are related by the BPS condition.

The method for constructing actions for supersymmetric D-branes is known in the literature $[5,6,7,8,9]$. These actions are obtained embedding the world-volume of the brane in superspace. The fermionic superspace coordinate becomes consequently a fermion on the brane. Apparently, this seems to imply that the brane breaks all the supersymme-

tries, since the fermion plays the role of the Goldstino field. The solution of this apparent paradox is the fact that the brane action possesses an additional local fermionic symmetry, known as $\kappa$-symmetry [10, 11], whose role is to decouple half of the fermions in the brane action. After $\kappa$-gauge fixing half of the supersymmetries become linearly realized, while the other half are still non linearly realized, a la Volkov-Akulov [12]. Therefore, $\kappa$-symmetry is a basic ingredient in the construction of brane actions, and it is the world-volume remnant of the BPS-condition.

Spacetime-filling D-branes characterize the vacua of type-I models. Type-I string theory is obtained from type-IIB through an orientifold projection [13] that removes the states that are odd under orientation reversal of the string. From a target space point of view, this can be pictured in terms of orientifold planes, and the appearance of tadpoles corresponds in this picture to non-vanishing tension and charge of the O-plane. Tadpole cancellation typically requires the introduction of an open sector, and this corresponds to D-branes. In ten dimensions, one can thus introduce an $O_{-}$-plane (with negative tension and negative charge) and 32 D9-branes, with a resulting gauge group $S O(32)$ [14]. The cancellation of the overall tension and charge of the configuration corresponds to the cancellation of 
dilaton and $\mathrm{RR}$ tadpoles $[15,16]$. The resulting theory is $\mathcal{N}=1$ supersymmetric, and the massless spectrum contains the gravity multiplet from the closed sector and an $S O(32)$ Yang-Mills multiplet from the open sector. There is actually a second possibility, corresponding to a change of sign of the tension and the charge of the orientifold plane, so that $\mathrm{RR}$ tadpole cancellation requires the addition of 32 anti-D9 branes, with a resulting gauge group $U S p(32)$ [17]. The overall tension of the configuration does not vanish, so that the resulting theory has a dilaton tadpole. Nevertheless, the theory is anomaly-free, as a consequence of the vanishing of the RR tadpole $[16,18]$. The spectrum is not supersymmetric, and more precisely the closed sector is not modified, still describing at the massless level the $\mathcal{N}=1$ gravity multiplet, while the massless fermions in the open sector are not in the adjoint but in the antisymmetric representation of $U S p(32)$, so that supersymmetry is broken on the brane [19]. The gravitino couplings can then only be consistent if supersymmetry is non-linearly realized in the open sector. Since the antisymmetric representation of symplectic groups is reducible, the massless spectrum contains a spinor that is an USp $(32)$ singlet, and this spinor is the goldstino of the non-linearly realized supersymmetry [20]. The presence of the NS tadpole is a manifestation of the fact that the theory has been expanded around the wrong vacuum, and an analysis of this problem, addressed long time ago in [21], has been recently performed in [22].

From the point of view of the low-energy effective action, the closed sector of type-I strings is obtained performing a consistent $Z_{2}$ truncation of the type-IIB theory, while the open sector corresponds to the first order in the low-energy expansion of the D9-brane action in a type-I background. It is then natural to ask what is the fate of $\kappa$-symmetry in this background. The result is that there are two possibilities of performing this truncation [23], and in a flat background, with all bulk fields put to zero, the D9-brane action reduces in one case to the Volkov-Akulov (VA) action [12], and in the other case to a constant. In [24] these results were extended to a generic background, showing that also in the curved case there are two possibilities of performing the truncation. In one case one gets a dilaton tadpole and a RR tadpole plus goldstino couplings, while in the other case the goldstino couplings vanish and one is left with a dilaton and a $R R$ tadpole. This result is equivalent to the string result: the two different truncations correspond to the two different choices of the relative sign of tension and charge of orientifold plane and D9-branes. The first case corresponds to the non-supersymmetric one, in which the orientifold plane and the D-brane have both positive tension, and in the case of 32 coincident D9-branes it gives rise to the 
low-energy action $[20,25]$ of the $U S p(32)$ model. The second case, in which the goldstino disappears, corresponds to an orientifold plane with negative tension, and in the case of 32 coincident D9-branes it gives rise to the low-energy action of the supersymmetric $S O(32)$ superstring. In other words, the supersymmetric truncation projects out the spinor that would not be projected out fixing the $\kappa$-symmetry gauge. As a result, in general one expects that only linearly realized supersymmetry survives. The non-supersymmetric truncation does the opposite, namely it projects out the spinor that would have been projected out by $\kappa$-symmetry, so that no $\kappa$-symmetry is left in the truncated theory, and in the resulting action supersymmetry is only non-linearly realized, i.e. completely broken.

If one wants to generalize these results to lower dimensional cases, the first possibility is to consider the T-dual of this configuration, that is a Type- $\mathrm{I}^{\prime}$ orientifold of type-IIA compactified on a circle [2]. In this case the orientifold projection has fixed points on the circle, corresponding to the positions of the O-planes. In [26] the low-energy action for a D8-brane located at one of the fixed points was constructed in the Type-I' background, without including the fermionic fields. In this paper we want to apply the techniques used in [24] to this case, in order to obtain the low-energy brane+bulk action up to four fermi terms, for a generic 9-dimensional background. We will see that the results are in complete agreement with T-duality, since also in this case one has two possible consistent truncations, leading in one case to a supersymmetric model, and in the other case to a model in which supersymmetry is non-linearly realized on the brane.

S-duality is a symmetry of type-IIB string theory mapping weak coupling to strong coupling [27]. On the other hand, type-I string theory is related in ten dimensions by a strong-weak coupling S-duality to the heterotic $S O(32)$ theory [28]. In this respect, it is interesting to study the behavior of the O9-D9 system of $[23,24]$ under S-duality, and whether the result can be related to the low-energy action of the heterotic theory. Type-IIB supersymmetry algebra includes two 10-forms [29, 23]. One of them is the RR 10-form that couples to D9-branes, while the other couples to other spacetime-filling branes, called NS9branes in [29]. Type-IIB supergravity also admits an additional $Z_{2}$ truncation, removing all the RR fields. This truncation was conjectured in [29] to be the S-dual of the orientifold projection, and consequently the introduction of 32 NS9-branes was conjectured to give origin to the $S O(32)$ heterotic string after performing this projection $[29,30]$. Under Sduality, the DBI part of the D9-brane action acquires a dilaton factor $e^{-4 \phi}$ in the string frame, and this led to conjecture that the tension of the NS9-branes is proportional to $g_{S}^{-4}$ 
$[29,31]$. We will argue in this paper that this is actually not the case. We will prove that $\kappa$-symmetry requires a dilation factor $e^{-2 \phi}$ in front of the DBI term of an NS9-brane action, and thus a tension proportional to $g_{S}^{-2}$. This seems to be inconsistent with the analysis of [31], since starting from a D9-brane action and performing an S-duality transformation one should end up with a $\kappa$-symmetric action. The solution of this paradox is that in the presence of NS and RR 10-forms S-duality is no longer a symmetry of the type-IIB algebra. This does not mean that S-duality symmetry of type-IIB is actually broken, since introducing spacetime-filling branes is only consistent after performing a truncation. We will also analyze the type-IIA case, since type-IIA supersymmetry algebra can be extended including an NS 10-form, and the resulting supersymmetric NS9-brane action is $\kappa$-symmetric if the tension scales like $e^{-2 \phi}$ in the string frame.

The paper is organized as follows. In section 2 we review some known results about super D-branes. In section 3 we discuss the type-I' truncation of type-IIA compactified on a circle. We make use of the "democratic formulation" of the theory [26], in which both the RR forms and their magnetic duals appear as independent fields in the supersymmetry algebra, and duality relations between electric and magnetic field strengths are imposed as constraints (the same formulation was introduced in [23] for the type-IIB case). We show that the two possible truncations lead in one case to a supersymmetric model, and in the other case to a model in which supersymmetry is spontaneously broken on the D8-brane. In section 4 we discuss S-duality of type-IIB in the presence of NS and RR 10-forms. First of all, one realizes that these two fields, besides transforming as a doublet under S-duality, acquire a dilaton dependence $e^{-2 \phi}$. Moreover, an additional constraint has to be imposed for S-duality to be a symmetry. In other words, S-duality is broken in the presence of spacetime-filling branes. Section 5 is devoted to the study of the supersymmetric NS9brane in both IIA and IIB. We show that $\kappa$-symmetry implies that the tension of the NS9-brane in the string frame scales like $e^{-2 \phi}$. In analogy to the D9 [24] and the D8 cases, one can perform a truncation to show that half of the fermions decouple from the spectrum. In this case the spectrum is projected by means of a heterotic truncation. Finally, section 6 contains the conclusions. 


\section{Generalities about D-brane actions}

In this section we review the basic ingredients for the construction of supersymmetric Dbranes, and in particular spacetime-filling D-branes. We will concentrate here on the IIB case, while the straightforward generalization to the IIA case will be outlined in the next section.

In order to construct supersymmetric actions for D-branes, one has to embed the Dbrane in IIB (or IIA) superspace. A basic ingredient is therefore the supersymmetry algebra of type IIB in 10 dimensions. Since we want a formulation that is suitable for all the Dbranes of type-IIB, we write down the IIB algebra in the democratic formulation, in which all the forms and their magnetic duals appear in the algebra. Following the notations of [23], the supersymmetry transformations of the IIB bulk fields are

$$
\begin{aligned}
& \delta e_{\mu}{ }^{a}=\bar{\epsilon} \Gamma^{a} \psi_{\mu} \\
& \delta \psi_{\mu}=D_{\mu} \epsilon-\frac{1}{8} H_{\mu \nu \rho} \Gamma^{\nu \rho} \sigma_{3} \epsilon+\frac{1}{16} e^{\phi} \sum_{n=0}^{5} \frac{1}{(2 n+1) !} G_{\mu_{1} \ldots \mu_{2 n+1}}^{(2 n+1)} \Gamma^{\mu_{1} \ldots \mu_{2 n+1}} \Gamma_{\mu} \mathcal{P}_{n} \epsilon \\
& \delta B_{\mu \nu}^{(2)}=2 \bar{\epsilon} \sigma_{3} \Gamma_{[\mu} \psi_{\nu]}, \\
& \delta B_{\mu_{1} \ldots \mu_{10}}^{(10)}=e^{-2 \phi} \bar{\epsilon} \sigma_{3}\left(10 \Gamma_{\left[\mu_{1} \ldots \mu_{9}\right.} \psi_{\left.\mu_{10}\right]}-\Gamma_{\mu_{1} \ldots \mu_{10}} \lambda\right), \\
& \delta C_{\mu_{1} \ldots \mu_{2 n}}^{(2 n)}=-(2 n) e^{-\phi} \bar{\epsilon} \mathcal{P}_{n} \Gamma_{\left[\mu_{1} \ldots \mu_{2 n-1}\right.}\left(\psi_{\left.\mu_{2 n}\right]}-\frac{1}{2(2 n)} \Gamma_{\left.\mu_{2 n}\right]} \lambda\right) \\
& \quad+n(2 n-1) C_{\left[\mu_{1} \ldots \mu_{2 n-2}\right.}^{(2 n-2)} \delta B_{\left.\mu_{2 n-1} \mu_{2 n}\right]}, \\
& \delta \lambda=\partial_{\mu} \phi \Gamma^{\mu} \epsilon-\frac{1}{12} H_{\mu \nu \rho} \sigma_{3} \Gamma^{\mu \nu \rho} \epsilon+\frac{1}{4} e^{\phi} \sum_{n=0}^{5} \frac{n-2}{(2 n+1) !} G_{\mu_{1} \ldots \mu_{2 n+1}}^{(2 n+1)} \mathcal{P}_{n} \Gamma^{\mu_{1} \ldots \mu_{2 n+1}} \epsilon \\
& \delta \phi=\frac{1}{2} \bar{\epsilon} \lambda \quad,
\end{aligned}
$$

where $\mathcal{P}_{n}$ is $\sigma_{1}$ for $n$ odd and $i \sigma_{2}$ for $n$ even. We are neglecting terms cubic in the fermions in the case of the transformations of the spinors. We have introduced the field strengths for the RR fields and their duals, related by duality according to the relations

$$
G^{(7)}=-* G^{(3)} \quad, \quad G^{(9)}=* G^{(1)} \quad, \quad G^{(5)}=* G^{(5)}
$$

An advantage of this formulation is that all the Chern-Simons terms in the supergravity lagrangian are hidden in the definitions of the field strengths and their magnetic duals. The matching between bosonic and fermionic degrees of freedom is of course restored only once 
these duality relations are imposed ${ }^{1}$. The field strengths are defined through the relations

$$
\begin{aligned}
& H=d B \\
& G^{(2 n+1)}=d C^{(2 n)}-H C^{(2 n-2)},
\end{aligned}
$$

and the gauge transformations of the fields are

$$
\begin{aligned}
& \delta B=d \Lambda_{N S}, \\
& \delta B^{(10)}=d \Lambda_{N S}^{(10)}, \\
& \delta C^{(2 n)}=d \Lambda_{R R}^{(2 n-1)}-\Lambda_{R R}^{(2 n-3)} H,
\end{aligned}
$$

so that the field strengths are gauge invariant. The dilaton dependence in the variations of the forms shows that the algebra of eq. (2.1) is expressed in the string frame. Moreover, it is important to observe that two 10-forms are present in the algebra. We stress again that, even though these forms do not have any dynamics since they do not have any field strength, they are associated to spacetime-filling branes, whose presence is consistent only after one performs a suitable projection of the spectrum.

The general idea is to describe supersymmetric D-branes through the embedding of a bosonic brane in superspace $[5,7,8]$. We thus introduce the world-volume fields as the supercoordinates

$$
Z^{M}\left(\xi^{i}\right)=\left(x^{\mu}\left(\xi^{i}\right), \theta^{\alpha I}\left(\xi^{i}\right)\right)
$$

defining the position of the brane in superspace. Here $\xi^{i}$ are the world-volume coordinates $(i=0, \ldots, 9$ for a 9 -brane), while $\mu=0, \ldots, 9$ is a spacetime vector index and $\alpha=1, \ldots, 32$ a spinor index, and $I=1,2$. The Majorana spinors $\theta^{I}$ are both left-handed ${ }^{2}$. We denote with $V^{i}(\xi)$ the abelian world-volume vector. The bulk superfields are denoted with

$$
\left\{\phi, E_{M}^{A}, B_{M N}, B_{M_{1} \ldots M_{10}}, C_{M_{1} \ldots M_{2 n}}^{(2 n)}\right\} \quad, \quad n=0, \ldots, 5
$$

and the brane action is

$$
S=S_{D B I}+S_{W Z}=-\int_{M_{10}} d^{10} \xi e^{-\phi} \sqrt{-\operatorname{det}(g+\mathcal{F})}+\int_{M_{10}} C e^{\mathcal{F}}
$$

\footnotetext{
${ }^{1}$ This is a generalization of what is typically done for the self-dual 5 -form field strength, when one writes a lagrangian for an ordinary 5 -form, and imposes self-duality as a constraint on the equations of motion.

${ }^{2}$ In the IIA case the two chiral spinors $\theta^{I}$ are substituted with a single non-chiral Majorana spinor.
} 
where

$$
\mathcal{F}_{i j}=F_{i j}+B_{i j}
$$

and one defines the pull-back of the bulk fields on the world-volume according to

$$
g_{i j}=E_{i}^{a} E_{j}^{b} \eta_{a b} \quad, \quad B_{i j}=\partial_{i} Z^{M} \partial_{j} Z^{N} B_{M N}
$$

and

$$
C=\sum_{n=0}^{5}(-1)^{n} C^{(2 n)} \quad, \quad C^{(2 n)}=\frac{1}{(2 n) !} d Z^{M_{1}} \ldots d Z^{M_{2 n}} C_{M_{1} \ldots M_{2 n}}^{(2 n)} .
$$

In a flat space background $[6,9]$ these expressions have a simpler form, since from the (global) supersymmetry transformations of the supercoordinates,

$$
\begin{aligned}
& \delta \theta=-\epsilon \\
& \delta x^{\mu}=\frac{1}{2}\left(\bar{\epsilon} \Gamma^{\mu} \theta\right)
\end{aligned}
$$

one derives a supersymmetry invariant object

$$
\Pi_{i}^{\mu}=\partial_{i} x^{\mu}+\frac{1}{2}\left(\bar{\theta} \Gamma^{\mu} \partial_{i} \theta\right)
$$

that is the flat space analogous of $\partial_{i} Z^{M} E_{M}{ }^{a}$. Consequently, the pull-back of the metric becomes

$$
g_{i j}=\Pi_{i}^{\mu} \Pi_{j}^{\nu} \eta_{\mu \nu}=\partial_{i} x^{\mu} \partial_{j} x^{\nu} \eta_{\mu \nu}+\partial_{(i} x^{\mu} \bar{\theta} \Gamma_{\mu} \partial_{j)} \theta+\ldots
$$

where we neglect higher terms in the fermions. Analogously, the pull-back of the NS 2-form is

$$
B_{i j}=\partial_{[i} x^{\mu} \bar{\theta} \sigma_{3} \Gamma_{\mu} \partial_{j]} \theta+\ldots
$$

while the pull-back of the RR forms is

$$
C_{i_{1} \ldots i_{2 n}}^{(2 n)}=-n e^{-\phi} \partial_{\left[i_{1}\right.} x^{\mu_{1}} \ldots \partial_{i_{2 n-1}} x^{\mu_{2 n-1}} \bar{\theta} \mathcal{P}_{n} \Gamma_{\mu_{1} \ldots \mu_{2 n-1}} \partial_{\left.i_{2 n}\right]} \theta+\ldots
$$

The brane action (2.7) is then supersymmetric, provided that one chooses the supersymmetry transformation for the world-volume vector $V_{i}$ to be

$$
\delta V_{i}=-\frac{1}{2} \bar{\epsilon} \gamma_{i} \sigma_{3} \theta-\frac{1}{2} \bar{\epsilon} \gamma^{j} \theta F_{j i}
$$

up to a gauge transformation.

The action (2.7) is invariant under world-volume general coordinate transformations, and one can then choose a static (or Monge) gauge, in which the coordinates $\xi^{i}$ are identified 
with $x^{i}, i=0, \ldots p$, where $p+1$ is the spacetime dimension of the brane. A supersymmetry variation then induces a compensating general coordinate transformation, and the resulting variation for $\theta$ is

$$
\delta \theta=-\epsilon-\frac{1}{2}\left(\bar{\epsilon} \Gamma^{i} \theta\right) \partial_{i} \theta
$$

The other $x$ 's in this gauge become world-volume scalars, whose supersymmetry transformations is

$$
\delta \phi^{a}=\frac{1}{2} \bar{\epsilon} \Gamma^{a} \theta-\frac{1}{2}\left(\bar{\epsilon} \Gamma^{i} \theta\right) \partial_{i} \phi^{a} \quad, \quad a=p+1, \ldots, 10
$$

Focusing again on the flat space limit, one can recognize in eq. (2.17) the Volkov-Akulov (VA) transformations [12]. We will concentrate in the following on space-filling 9-branes, so that the target spacetime $\Gamma$-matrices can be identified with the world-volume $\gamma$-matrices, and the spacetime index $\mu$ is the same as the world-volume index $i$. The commutator of two transformations (2.17) is a translation,

$$
\left[\delta_{1}, \delta_{2}\right] \theta=\left(\bar{\epsilon}_{2} \gamma^{\mu} \epsilon_{1}\right) \partial_{\mu} \theta
$$

and thus eq. (2.17) provides a realization of supersymmetry. The 1-form

$$
e_{\mu}^{a}=\delta_{\mu}^{a}+\frac{1}{2}\left(\bar{\theta} \gamma^{a} \partial_{\mu} \theta\right)
$$

transforms under supersymmetry as

$$
\delta e^{a}=L_{\xi} e^{a}
$$

with $L_{\xi}$ the Lie derivative with respect to ${ }^{3}$

$$
\xi_{\mu}=-\frac{1}{2}\left(\bar{\epsilon} \gamma_{\mu} \theta\right)
$$

The action of supersymmetry on $e$ is thus a general coordinate transformation, with a parameter depending on $\theta$, and therefore

$$
\mathcal{L}=-\operatorname{det} e
$$

is clearly an invariant Lagrangian. Using the same technique, for a generic field $A$ that transforms under supersymmetry as

$$
\delta A=L_{\xi} A
$$

\footnotetext{
${ }^{3}$ The parameter $\xi$ should not be confused with the world-volume coordinates.
} 
defining the induced metric as $g_{\mu \nu}=e_{\mu}{ }^{m} e_{\nu m}$, a supersymmetric lagrangian in flat space is determined by the substitution

$$
\mathcal{L}(\eta, A) \rightarrow e \mathcal{L}(g, A)
$$

This is what happens in the brane action (2.7) in the Monge gauge in a flat space background, since the pull-back of the metric of eq. (2.13) in the Monge gauge equals the VA metric $g_{\mu \nu}$. Moreover, the second term in the variation of $V^{i}$ is a general coordinate transformation with the same parameter $\xi$ plus an additional gauge transformation, while the first term combines with the variation of the pull-back of the NS form of eq. (2.14) in such a way that $\mathcal{F}$ transforms covariantly. Finally, the pull-backs of the RR forms in eq. (2.15) are such that the WZ term in the brane action transforms as a total derivative.

It is then natural to generalize this VA construction to D9-branes in a generic background. One must construct from the bulk fields quantities whose supersymmetry variations are general coordinate transformations with the parameter $\xi$ plus additional gauge transformations [20, 25]. Supersymmetry guarantees that this way of constructing the D9brane action coincides with the superspace construction of $[7,8]^{4}$. For instance, from the supersymmetry variation of $\phi$ one defines

$$
\begin{aligned}
\hat{\phi}= & \phi+\frac{1}{2} \bar{\theta} \lambda-\frac{1}{48} H_{i j k} \bar{\theta} \gamma^{i j k} \sigma_{3} \theta \\
& +\frac{1}{16} e^{\phi} \sum_{n=1}^{6} \frac{n-3}{(2 n-1) !} G_{i_{1} \ldots i_{2 n-1}}^{(2 n-1)} \bar{\theta} \gamma^{i_{1} \ldots i_{2 n-1}} \mathcal{P}_{n} \theta
\end{aligned}
$$

whose supersymmetry transformation is a general coordinate transformation with the correct parameter $\xi_{i}$ given in (2.22), up to higher order fermi terms. With the same technique, one can construct all the other hatted fields [20, 25], so that the resulting D9-brane action in a generic type-IIB background is

$$
S=S_{D B I}+S_{W Z}=-\int_{M_{10}} d^{10} \xi e^{-\hat{\phi}} \sqrt{-\operatorname{det}(\hat{g}+\mathcal{F})}+\int_{M_{10}} \hat{C} e^{\mathcal{F}}
$$

where

$$
\mathcal{F}_{i j}=F_{i j}+\hat{B}_{i j}
$$

We come now to a brief discussion of the degrees of freedom that the action (2.7) propagates. If all the fermions $\theta$ were dynamical this would lead to a complete spontaneous

\footnotetext{
${ }^{4}$ See [32] for a similar construction in the case of a generic p-brane.
} 
supersymmetry breaking, since the $\theta$ 's transform non-linearly under supersymmetry. It is well known that this is actually not the case because of $\kappa$-symmetry gauge invariance, whose fixing leads to a cancellation between the DBI and the WZ term that makes only half of the fermions propagate. To leading order in the fermions, and in the Monge gauge, the $\kappa$-symmetry transformation for $\theta$ and $V_{i}$ reads

$$
\begin{aligned}
& \delta \theta=\frac{1}{2}\left(1-\sigma_{1}-\frac{i}{2} \sigma_{2} F^{i j} \Gamma_{i j}\right) \kappa+\ldots \\
& \delta V_{i}=-\frac{1}{2} \delta \bar{\theta} \gamma_{i} \sigma_{3} \theta
\end{aligned}
$$

with $\kappa$ an $S L(2, R)$ doublet of spinors, and neglecting higher order terms in $\theta$ and $F$ in the variation of $\theta$. This gauge invariance can be used to put $\theta_{1}-\theta_{2}=0$. After a supersymmetry transformation, this gauge choice is maintained through a compensating $\kappa$-transformation of parameter $\kappa_{1}-\kappa_{2}=\epsilon_{1}-\epsilon_{2}$, and this results in the linear supersymmetry transformations

$$
\begin{aligned}
& \delta\left(\theta_{1}+\theta_{2}\right)=\frac{1}{4} F^{i j} \Gamma_{i j}\left(\epsilon_{1}-\epsilon_{2}\right) \\
& \delta V_{i}=-\frac{1}{2}\left(\bar{\epsilon}_{1}-\bar{\epsilon}_{2}\right) \Gamma_{i}\left(\theta_{1}+\theta_{2}\right)
\end{aligned}
$$

and expanding the DBI action with this gauge choice one obtains that these are the correct linear supersymmetry transformations $[9,33]^{5}$. In other words, $\kappa$ symmetry is the brane effective action equivalent of the statement that a brane solution of supergravity is a BPS solution preserving half of the supersymmetries. In the case of spacetime-filling branes, that do not correspond to any solution of supergravity, we assume in this paper that $\kappa$-symmetry is the only requirement that these branes have to satisfy.

At the end of this section, we now want to review the results of [23] and [24]. One can perform a type-I truncation of IIB supersymmetry algebra, imposing

$$
\begin{aligned}
& C^{(2 n-2)}=0, \quad n=1,3,5, \\
& B=0, \\
& B^{(10)}=0, \\
& \left(1 \pm \sigma_{1}\right) f=0,
\end{aligned}
$$

where we have denoted with $f$ the gravitino and the dilatino. The surviving bosonic fields are thus the dilaton, the metric, the RR 2-form and its dual, and the RR 10-form, while

\footnotetext{
${ }^{5}$ See $[34]$ for a similar analysis.
} 
the two different signs in the projection of the fermions indicate that there are two possible type-I truncations.

The truncation on the D9-brane action was performed in [23] in flat space, and generalized in [24] to an arbitrary background. We review here the results. The brane fields are projected according to

$$
\begin{aligned}
& V_{i}=0, \\
& \left(1 \pm \sigma_{1}\right) \theta=0
\end{aligned}
$$

The lower sign choice leads to no surviving $\kappa$-symmetry, since it projects out the spinor components that would have been put to zero using $\kappa$-symmetry before the truncation, while the upper sign choice leads to no leftover components of $\theta$. This last choice, then, corresponding to the vanishing of all the terms containing the goldstino, results in a supersymmetric type-I spectrum. Actually, in the case of a single D9-brane, there are no remaining world-volume degrees of freedom after the truncation, but the generalization to a stuck of branes would result in a spectrum in which supersymmetry is linearly realized, and the goldstino is projected out. The resulting action contains a dilaton tadpole and a RR tadpole, that in the $S O(32)$ string are both canceled against the orientifold plane contribution. The other choice, instead, corresponds to the curved generalization of the VA action. The resulting spectrum breaks supersymmetry in the brane sector [19], or more precisely $\mathcal{N}=1$ supersymmetry is non-linearly realized on the brane. The brane action again contains a dilaton tadpole and a RR tadpole, but in this case, a suitable orientifold projection only cancels the brane $\mathrm{RR}$ charge, and a dilaton tadpole remains [17].

The type-IIB supersymmetry algebra in $\mathrm{D}=10$ also admits an alternative $Z_{2}$ truncation, projecting out all the RR fields and acting as $\left(1 \pm \sigma_{3}\right) f=0$ on the fermions, and for this reason called 'heterotic truncation' [23]. We will show in section 5 how this truncation can be consistently implemented on spacetime-filling branes electrically charged with respect to $B^{(10)}$, after a discussion about S-duality of type-IIB carried out in section 4 . First, in the next section, we are going to describe the T-duals of these results, i.e. the type- $\mathrm{I}^{\prime}$ truncation of IIA in the presence of D8-branes. 


\section{$3 \quad$ Type-I' Truncation of IIA}

After reduction to $\mathrm{D}=9$, T-duality relates the system described in the previous section to type-IIA theory compactified on a dual circle, and the corresponding truncation is in this case the low-energy manifestation of the type-I' orientifold projection. In this section we want to discuss this truncation in the presence of D8-branes. Since D8-branes are charged with respect to the RR 9-form, whose field strength is dual to a cosmological constant, the massive Romans IIA supergravity [35] is the bulk low-energy theory describing this system [28]. In has been shown in [26] that both massless and massive 10-dimensional IIA supergravities can be described in terms of the same supersymmetry algebra, once the Romans cosmological constant is treated as a dynamical 0-form dual to the RR 10-form field strength. Again, it is convenient to work in the democratic formulation [23, 26], treating all the RR-forms and their magnetic duals as independent, and imposing the duality relations as constraints. The resulting supersymmetry algebra is

$$
\begin{aligned}
& \delta e_{\mu}{ }^{a}=\bar{\epsilon} \Gamma^{a} \psi_{\mu}, \\
& \delta \psi_{\mu}=D_{\mu} \epsilon+\frac{1}{8} H_{\mu \nu \rho} \Gamma^{\nu \rho} \Gamma_{11} \epsilon+\frac{1}{16} e^{\phi} \sum_{n=0}^{5} \frac{1}{(2 n) !} G_{\mu_{1} \ldots \mu_{2 n}}^{(2 n)} \Gamma^{\mu_{1} \ldots \mu_{2 n}} \Gamma_{\mu}\left(\Gamma_{11}\right)^{n} \epsilon, \\
& \delta B_{\mu \nu}^{(2)}=2 \bar{\epsilon} \Gamma_{11} \Gamma_{[\mu} \psi_{\nu]}, \\
& \delta C_{\mu_{1} \ldots \mu_{2 n-1}}^{(2 n-1)}=-(2 n-1) e^{-\phi} \bar{\epsilon}\left(\Gamma_{11}\right)^{n} \Gamma_{\left[\mu_{1} \ldots \mu_{2 n-2}\right.}\left(\psi_{\left.\mu_{2 n-1}\right]}-\frac{1}{2(2 n-1)} \Gamma_{\left.\mu_{2 n-1}\right]} \lambda\right) \\
& \quad+(n-1)(2 n-1) C_{\left[\mu_{1} \ldots \mu_{2 n-3}\right.}^{(2 n-3)} \delta B_{\left.\mu_{2 n-2} \mu_{2 n-1}\right]}, \\
& \delta \lambda=\partial_{\mu} \phi \Gamma^{\mu} \epsilon-\frac{1}{12} H_{\mu \nu \rho} \Gamma_{11} \Gamma^{\mu \nu \rho} \epsilon+\frac{1}{8} e^{\phi} \sum_{n=0}^{5} \frac{5-2 n}{(2 n) !} G_{\mu_{1} \ldots \mu_{2 n}}^{(2 n)}\left(\Gamma_{11}\right)^{n} \Gamma^{\mu_{1} \ldots \mu_{2 n}} \epsilon, \\
& \delta \phi=\frac{1}{2} \bar{\epsilon} \lambda .
\end{aligned}
$$

The RR field strengths are defined as

$$
G^{(2 n)}=d C^{(2 n-1)}-d B^{(2)} \wedge C^{(2 n-3)}+G^{(0)} e^{B^{(2)}},
$$

where it is understood that one has to extract the $2 n$-form out of $e^{B^{(2)}}$, and they are related by the duality relations

$$
G^{(2 n)}=(-)^{n} \star G^{(10-2 n)} .
$$

The field equations of type-IIA supergravity obtained in this formulation are supersymmetric only after these duality relations are imposed. This algebra can be naturally extended 
to include a 10-form, whose supersymmetry transformation is

$$
\delta B_{\mu_{1} \ldots \mu_{10}}^{(10)}=e^{-2 \phi}\left(-10 \bar{\epsilon} \Gamma_{\left[\mu_{1} \ldots \mu_{9}\right.} \psi_{\left.\mu_{10}\right]}+\bar{\epsilon} \Gamma_{\mu_{1} \ldots m_{10}} \lambda\right)
$$

Actually, there is also another consistent 10-form, whose transformation is

$$
\delta B_{\mu_{1} \ldots \mu_{10}}^{(10)}=e^{-2 \phi}\left(-10 \bar{\epsilon} \Gamma_{\left[\mu_{1} \ldots \mu_{9}\right.} \Gamma_{11} \psi_{\left.\mu_{10}\right]}-\bar{\epsilon} \Gamma_{\mu_{1} \ldots m_{10}} \Gamma_{11} \lambda\right)
$$

but we will show that it does not correspond to any spacetime-filling $\kappa$-symmetric brane. Consequently, we will only consider (3.4) as a natural extension of the type-IIA supersymmetry algebra.

We now continue reviewing the results of [26] concerning the possible consistent $Z_{2}$ truncations of the algebra of eqs. (3.1) and (3.4). In 10 dimensions, only a single truncation is available, projecting out all the RR fields, and acting on the fermions as

$$
\left\{\psi_{\mu}, \lambda, \epsilon\right\} \rightarrow \pm \Gamma_{11}\left\{\psi_{\mu},-\lambda, \epsilon\right\}
$$

We will construct in section 5 the resulting $\kappa$-symmetric spacetime-filling brane, for which this truncation is consistent in the way described in the previous section. It will turn out that this brane is electrically charged with respect to the 10 -form $B^{(10)}$.

If we compactify the theory on a circle $S^{1}$, the resulting theory admits another $Z_{2}$ truncation, acting on the compactified coordinate as

$$
x^{9} \rightarrow-x^{9} \quad,
$$

thus acting as an orbifold projection, being the low-energy manifestation of the orientifold projection generated by introducing two orientifold 8-planes at the fixed points. If we only consider spacetime indices in the uncompactified directions, the projection acts on the fields according to

$$
\begin{aligned}
& \left\{g_{\mu \nu}, \phi, B_{\mu \nu}^{(2)}\right\} \rightarrow\left\{g_{\mu \nu}, \phi,-B_{\mu \nu}^{(2)}\right\} \\
& C_{\mu_{1} \ldots \mu_{2 n-1}}^{(2 n-1)} \rightarrow(-)^{n+1} C_{\mu_{1} \ldots \mu_{2 n-1}}^{(2 n-1)} \\
& \left\{\psi_{\mu}, \lambda, \epsilon\right\} \rightarrow \mp \Gamma^{9}\left\{\psi_{\mu},-\lambda, \epsilon\right\}
\end{aligned}
$$

Any index in the 9-direction corresponds to an additional minus sign with respect to these projection rules, and consequently the 10 -form $B^{(10)}$ of eq. (3.4) (having an index in the 
9-direction) is consistently projected out $^{6}$. In order to make the analogy with the IIB case in 10 dimensions manifest, we define the 10-dimensional $\Gamma$-matrices as

$$
\begin{aligned}
& \Gamma^{\mu}=\gamma^{\mu} \otimes \sigma_{2}, \\
& \Gamma^{9}=\mathbf{1} \otimes \sigma_{1}, \\
& \Gamma_{11}=\mathbf{1} \otimes \sigma_{3}
\end{aligned}
$$

in terms of the 9-dimensional $\gamma$-matrices. Consequently, denoting the 10-dimensional IIA spinors as doublets of 9-dimensional spinors, the truncation acts as

$$
\begin{aligned}
& \left(1 \pm \sigma_{1}\right) \psi_{\mu}=0 \\
& \left(1 \mp \sigma_{1}\right) \lambda=0
\end{aligned}
$$

We now want to consider the introduction of D8-branes, and we will only take into account the case in which a single D8-brane is located at one of the two fixed points of the orientifold projection. Consistency requires that the truncation acts on the world-volume fields as ${ }^{7}$

$$
\begin{aligned}
& V^{i}=0, \\
& \left(1 \pm \sigma_{1}\right) \theta=0
\end{aligned}
$$

The brane action contains, in a massive background, an additional Chern-Simons term $[36,37]$ that we will not take into account because it vanishes after the truncation. The relevant terms in the brane action are thus

$$
-\int_{M_{9}} e^{-\phi} \sqrt{-\operatorname{det} g}+\int_{M_{9}} C^{(9)} .
$$

The supersymmetrization of this action is obtained in the Monge gauge following the same arguments of the previous section. Taking into account only the terms that are relevant after the truncation, and neglecting higher order fermi fields, we thus define the hatted fields

$$
\begin{aligned}
& \hat{\phi}=\phi+\frac{1}{2} \bar{\theta} \lambda+\ldots, \\
& \hat{g}_{\mu \nu}=g_{\mu \nu}+2 \bar{\theta} \gamma_{(\mu} \psi_{\nu)}+\bar{\theta} \gamma_{(\mu} D_{\nu)} \theta+\ldots, \\
& \hat{C}_{\mu_{1} \ldots \mu_{9}}^{(9)}=C_{\mu_{1} \ldots \mu_{9}}^{(9)}-9 e^{-\phi} \bar{\theta} \Gamma_{\left[\mu_{1} \ldots \mu_{8}\right.} \Gamma_{11} \psi_{\left.\mu_{9}\right]}-\frac{1}{2} e^{-\phi} \bar{\theta} \Gamma_{\mu_{1} \ldots \mu_{9}} \Gamma_{11} \lambda \\
& \quad-\frac{9}{2} e^{-\phi} \bar{\theta} \Gamma_{\left[\mu_{1} \ldots \mu_{8}\right.} \Gamma_{11} D_{\left.\mu_{9}\right]} \theta+\ldots,
\end{aligned}
$$

\footnotetext{
${ }^{6}$ In the case of $B^{\prime(10)}$ defined in eq. (3.5), consistency would require that this form survive the projection.

${ }^{7}$ The other world-volume field, the scalar $x^{9}$, is of course projected out because of eq. (3.7).
} 
whose supersymmetry transformation has the form of a $\theta$-dependent general coordinate transformation (plus an additional gauge transformation in the case of the 9-form). Expressing then the brane action in terms of these hatted fields, it turns out that if one chooses the upper sign in the projection of the fermions, all the terms containing the goldstino $\theta$ disappear in the action, while the lower sign choice leads to an action of the VA type for $\theta$. We interpret this result in the same way as we did for the IIB case in 10 dimensions. The upper sign choice corresponds to a supersymmetric spectrum. Again, just as in the case of a single D9-brane, for a single D8-brane there are no remaining worldvolume degrees of freedom after the truncation, but the generalization to a stuck of branes would result in a spectrum in which supersymmetry is linearly realized, and the goldstino is projected out. The resulting action contains a dilaton tadpole and a RR tadpole, that in consistent supersymmetric orientifold models are both canceled against the orientifold plane contribution. The other choice, instead, corresponds to the case in which the brane and the orientifold plane have both positive tension. Consequently, $\mathcal{N}=1$ supersymmetry is non-linearly realized on the brane, and a suitable orientifold projection only cancels the brane RR charge, while a dilaton tadpole remains. The fact that this result is in agreement with the IIB result of refs. [23, 24] is a manifestation of T-duality [36, 38].

\section{S-duality of Type-IIB}

Type-IIB superstring theory is conjectured to be invariant under $S L(2, Z)$ transformations [27], a discrete subgroup of the isometry group $S L(2, R)$ of type-IIB supergravity [39]. This group acts on the complex scalar

$$
\tau=C_{0}+i e^{-\phi}
$$

as

$$
\tau \rightarrow \frac{a \tau+b}{c \tau+d}
$$

where

$$
\left(\begin{array}{ll}
a & b \\
c & d
\end{array}\right) \in S L(2, R)
$$

while the 2-forms $B^{(2)}$ and $C^{(2)}$ transform as a doublet. The matrix

$$
S=\left(\begin{array}{cc}
0 & 1 \\
-1 & 0
\end{array}\right)
$$


generates the S-duality transformation $\tau \rightarrow-\frac{1}{\tau}$, that for a vanishing axion background corresponds to $\phi \rightarrow-\phi$, and in type-IIB string theory this results in mapping weak coupling to strong coupling. $S L(2, Z)$ symmetry thus implies a strong-weak coupling self-duality of type-IIB string theory. Since $S$ maps $B^{(2)}$ to $C^{(2)}$ and viceversa, the duality interchanges the fundamental string and the NS5-brane with the D1-string and the D5-brane. We want to study here how spacetime-filling branes transform under S-duality, and since the 10forms $B^{(10)}$ and $C^{(10)}$ cannot appear consistently in the low-energy effective action, the only way of deducing their behavior under an $S$-transformation is to make use of the supersymmetry algebra. In the remaining of this section, we thus study how a transformation $S$ acts on the supersymmetry algebra (2.1).

In the string frame, S-duality acts on the metric as

$$
g_{\mu \nu} \rightarrow e^{-\phi} g_{\mu \nu}
$$

Because of the explicit dilaton dependence of this transformation, it is easier to consider a configuration with vanishing axion background. This is what we will do in the following, and it is understood that our results do not depend on this assumption. For completeness, we write again the IIB supersymmetry transformations in the string frame in this background:

$$
\begin{aligned}
& \delta e_{\mu}{ }^{a}=\bar{\epsilon} \Gamma^{a} \psi_{\mu} \\
& \delta \psi_{\mu}=D_{\mu} \epsilon-\frac{1}{8} H_{\mu \nu \rho} \Gamma^{\nu \rho} \sigma_{3} \epsilon+\frac{1}{48} e^{\phi} G_{\mu_{1} \mu_{2} \mu_{3}}^{(3)} \Gamma^{\mu_{1} \mu_{2} \mu_{3}} \Gamma_{\mu} \sigma_{1} \epsilon, \\
& \delta B_{\mu \nu}^{(2)}=2 \bar{\epsilon} \sigma_{3} \Gamma_{[\mu} \psi_{\nu]},
\end{aligned}
$$

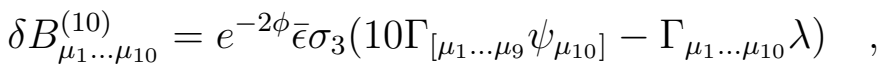

$$
\begin{aligned}
& \delta C_{\mu \nu}^{(2)}=-2 e^{-\phi} \bar{\epsilon} \sigma_{1} \Gamma_{[m}\left(\psi_{\nu]}-\frac{1}{4} \Gamma_{\nu]} \lambda\right) \\
& \delta C_{\mu_{1} \ldots \mu_{10}}^{(10)}=-10 e^{-\phi} \bar{\epsilon} \sigma_{1} \Gamma_{\left[\mu_{1} \ldots \mu_{9}\right.}\left(\psi_{\left.\mu_{10}\right]}-\frac{1}{20} \Gamma_{\left.\mu_{10}\right]} \lambda\right) \\
& \delta \lambda=\partial_{\mu} \phi \Gamma^{\mu} \epsilon-\frac{1}{12} H_{\mu \nu \rho} \sigma_{3} \Gamma^{\mu \nu \rho} \epsilon-\frac{1}{12} e^{\phi} G_{\mu \nu \rho}^{(3)} \sigma_{1} \Gamma^{\mu \nu \rho} \epsilon \quad, \\
& \delta \phi=\frac{1}{2} \bar{\epsilon} \lambda
\end{aligned}
$$

again neglecting higher order fermi terms in the transformations of the fermions.

Our strategy will be to derive the transformations of the fields under S-duality requiring 
that the supersymmetry algebra is preserved. We already know that

$$
\begin{aligned}
& \phi \rightarrow-\phi \\
& e_{\mu}{ }^{a} \rightarrow e^{-\phi / 2} e_{\mu}{ }^{a} .
\end{aligned}
$$

We now obtain the transformations of $\psi_{\mu}, \lambda$ and $e$ requiring that they are consistent with eqs. (4.7), i.e. imposing that the supersymmetry variation of the S-transformed fields is still eq. (4.6), up to other local symmetry transformations of the type-IIB theory. We know from the supersymmetry transformation of $\psi_{\mu}$ that $\epsilon$ and $\psi_{\mu}$ must acquire the same dilaton dependence. Moreover, we expect that all the fermions undergo an overall $S L(2, R)$ rotation determined by a $2 \times 2$ unitary matrix $\Omega$. Finally, the transformation of the gravitino can contain a term proportional to $\Gamma_{\mu} \lambda$. Hence, imposing that the transformed vielbein has the correct supersymmetry variation, one gets

$$
\begin{aligned}
& \epsilon \rightarrow e^{-\phi / 4} \Omega \epsilon \\
& \psi_{\mu} \rightarrow e^{-\phi / 4} \Omega \psi_{\mu}-\frac{1}{4} e^{-\phi / 4} \Omega \Gamma_{\mu} \lambda \quad, \quad
\end{aligned}
$$

It turns out that the supersymmetry transformation of the vielbein is mapped to itself plus an additional local Lorentz transformation of parameter

$$
\Lambda^{a b}=\frac{1}{4} e^{-\phi / 2}\left(\bar{\epsilon} \Gamma^{a b} \lambda\right)
$$

We neglect this term when we study the S-transformations of the supersymmetry variations of the fermions, since they would lead to cubic fermi terms. The S-duality transformation of $\lambda$ is straightforwardly obtained imposing that the transformed dilaton varies according to (4.6) under supersymmetry, and the result is

$$
\lambda \rightarrow-e^{\phi / 4} \Omega \lambda
$$

Proceeding this way, one realizes that the two 2-forms $B^{(2)}$ and $C^{(2)}$ form an $S L(2, R)$ doublet, transforming as

$$
\begin{aligned}
& B^{(2)} \rightarrow C^{(2)} \\
& C^{(2)} \rightarrow-B^{(2)}
\end{aligned}
$$

if

$$
\begin{aligned}
& \Omega^{-1} \sigma_{3} \Omega=-\sigma_{1}, \\
& \Omega^{-1} \sigma_{1} \Omega=\sigma_{3}
\end{aligned}
$$


whose solution is ${ }^{8}$

$$
\Omega=e^{-i \pi \sigma_{2} / 4}=\left(\begin{array}{cc}
1 / \sqrt{2} & -1 / \sqrt{2} \\
1 / \sqrt{2} & 1 / \sqrt{2}
\end{array}\right) .
$$

Implementing these transformations on the supersymmetry variation of $C^{(4)}$, one then obtains

$$
C_{\mu_{1} \ldots \mu_{4}}^{(4)} \rightarrow C_{\mu_{1} \ldots \mu_{4}}^{(4)}-6 B_{\left[\mu_{1} \mu_{2}\right.}^{(2)} C_{\left.\mu_{3} \mu_{4}\right]}^{(2)}
$$

leaving invariant the field strength

$$
G^{(5)}=d C^{(4)}-H^{(3)} \wedge C^{(2)} .
$$

The correctness of the transformations (4.8) and (4.10) is finally proven by showing that the supersymmetry variation of the transformed fermi fields is consistent with eqs. (4.6).

Following the same arguments, one can now determine the S-duality transformations of the two 10 -forms $B^{(10)}$ and $C^{(10)}$. One expects these fields to transform as a doublet, but the surprising result is that the first requirement one has to make to impose S-duality is that the transformation of this doublet must have a non-trivial dilaton dependence. More precisely, the only possibly consistent transformation is

$$
\begin{aligned}
& B^{(10)} \rightarrow e^{-2 \phi} C^{(10)}, \\
& C^{(10)} \rightarrow-e^{-2 \phi} B^{(10)}
\end{aligned}
$$

This still does not guarantee that S-duality is preserved, and in fact the additional variation of $\phi$ in (4.16) is canceled only once one imposes the additional constraints ${ }^{9}$

$$
\begin{aligned}
& C_{\mu_{1} \ldots \mu_{10}}^{(10)}(\bar{\epsilon} \lambda)=-e^{-\phi}\left(\bar{\epsilon} \sigma_{1} \Gamma_{\mu_{1} \ldots \mu_{10}} \lambda\right) \\
& B_{\mu_{1} \ldots \mu_{10}}^{(10)}(\bar{\epsilon} \lambda)=e^{-2 \phi}\left(\bar{\epsilon} \sigma_{3} \Gamma_{\mu_{1} \ldots \mu_{10}} \lambda\right)
\end{aligned}
$$

After performing the type-I truncation of eq. (2.31), in which $B^{(10)}$ is projected out, the first constraint becomes

$$
\frac{1}{10 !} \epsilon^{\mu_{1} \ldots \mu_{10}} C_{\mu_{1} \ldots \mu_{10}}=\mp e^{-\phi} \sqrt{-\operatorname{det} g}
$$

A similar result holds for the second constraint, after performing the heterotic truncation, in which all the RR fields are projected out and the spinors are projected according to

$$
\left(1 \pm \sigma_{3}\right) f=0
$$

\footnotetext{
${ }^{8}$ The inverse choice for $\Omega$ corresponds to a sign change in the transformations of $C^{(2)}$ and $B^{(2)}$.

${ }^{9}$ As a consistency check, one can show that these constraints are related by an S-duality transformation.
} 
As we will see in the next section, a possible interpretation of this result is that S-duality is actually broken in the presence of spacetime-filling branes. The constraint of eq. (4.17) can be justified only in the truncated theory, and this is in agreement with the fact that only in the truncated theory the presence of spacetime-filling branes can be consistent.

We will see in the next section that if one tries to construct the S-dual of a supersymmetric D9-brane using eqs. (4.7), (4.8), (4.10), (4.11) and (4.16), the action one gets is no longer $\kappa$-symmetric, and the constraints of eq. (4.17) have to be imposed to restore $\kappa$-symmetry. This means that the breakdown of $\kappa$-symmetry is consistent with the breakdown of S-duality. The brane action obtained in this way has a DBI term proportional to $e^{-4 \phi}$ and a WZ term proportional to $e^{-2 \phi}$. As we are going to prove, it turns out that, in the untruncated theory, a $\kappa$-symmetric action for a spacetime-filling brane charged with respect to $B^{(10)}$ has an $e^{-2 \phi}$ dilaton dependence in the DBI term, and no dilaton factor in the WZ term.

\section{$5 \quad$ Spacetime-filling branes and S-duality}

In this section we want to describe the $\kappa$-symmetric spacetime-filling branes that are charged with respect to the NS 10-forms of IIB and IIA supergravities. We start from the type-IIB case, performing an S-duality transformation on the D9-brane action. In the following of this section, we will always have in mind to perform a $Z_{2}$ truncation that leaves the NS 10-form invariant. In the case of IIB, this transformation projects out all the RR fields, leaving the NS fields invariant. This projection can actually be worked out using the results of the previous section, performing an S-duality transformation of the truncations of eq. (2.31). In the fermionic sector, performing the transformations (4.8) and (4.10) and using eq. (4.12), the projection becomes

$$
\left(1 \pm \sigma_{3}\right) f=0 \quad .
$$

The same projection applies to the spinor $\theta$ in the brane sector, since $\theta$ transforms under S-duality like $\epsilon$, while the world-volume vector $V^{i}$ is projected out, again in agreement with eqs. (4.8) and (4.12). We will assume that the 10 -forms $B^{(10)}$ and $C^{(10)}$ transform according to eq. (4.16), keeping in mind that this is consistent only if the constraints (4.17) are satisfied. We will see that the action we end up with is consistently $\kappa$-symmetric only if these constraints are satisfied. 
After the projection, the S-dual of the action (2.7) becomes

$$
S=-\int_{M_{10}} d^{10} \xi e^{-4 \phi} \sqrt{-\operatorname{det} g}+\int_{M_{10}} e^{-2 \phi} B^{(10)} .
$$

The supersymmetrization of this action is then worked out in the same way as the D9 and D8 cases. One first constructs the hatted fields

$$
\begin{aligned}
& \hat{\phi}=\phi+\frac{1}{2} \bar{\theta} \lambda+\ldots \quad, \\
& \hat{g}_{\mu \nu}=g_{\mu \nu}+2 \bar{\theta} \gamma_{(\mu} \psi_{\nu)}+\bar{\theta} \gamma_{(\mu} D_{\nu)} \theta+\ldots \quad, \\
& \hat{B}_{\mu_{1} \ldots \mu_{10}}^{(10)}=B_{\mu_{1} \ldots \mu_{10}}^{(10)}+10 e^{-2 \phi} \bar{\theta} \Gamma_{\left[\mu_{1} \ldots \mu_{9}\right.} \sigma_{3} \psi_{\left.\mu_{10}\right]}-e^{-2 \phi} \bar{\theta} \Gamma_{\mu_{1} \ldots \mu_{10}} \sigma_{3} \lambda \\
& \quad+5 e^{-2 \phi} \bar{\theta} \Gamma_{\left[\mu_{1} \ldots \mu_{9}\right.} \sigma_{3} D_{\left.\mu_{10}\right]} \theta+\ldots \quad,
\end{aligned}
$$

and then writes the supersymmetric action

$$
S=-\int_{M_{10}} d^{10} \xi e^{-4 \hat{\phi}} \sqrt{-\operatorname{det} \hat{g}}+\int_{M_{10}} e^{-2 \hat{\phi}} \hat{B}^{(10)} .
$$

If this procedure preserved $\kappa$-symmetry, it would be expected that one of the two truncations (the one with the upper sign choice in eq. (5.1), as one would get using eq. (4.12)) leaded to a brane action in which all the goldstino terms disappear. This is actually not the case, since for the upper sign choice a term proportional to

$$
B_{\mu_{1} \ldots \mu_{10}}^{(10)}(\bar{\epsilon} \lambda)+e^{-2 \phi}\left(\bar{\epsilon} \Gamma_{\mu_{1} \ldots \mu_{10}} \lambda\right)
$$

survives. This term vanishes if the second constraint of eq. (4.17) is imposed. This is not surprising, since only if this constraint is valid the S-duality transformations can be performed. Thus, the picture that emerges is that the breakdown of S-duality is in agreement with the breakdown of $\kappa$-symmetry, and the constraint of eq. (4.17) provides a restoration of both. On the other hand, the constraint (4.17) leads to a vanishing action for the NS9-brane, and this could simply mean that such an object does not exist. We will comment about this in the conclusions. The lower sign choice in (5.1), again analogously to the D-brane case, corresponds to a VA-type action for $\theta$, after eq. (4.17) is imposed.

Let us consider now the action

$$
S=-\int_{M_{10}} d^{10} \xi e^{-2 \hat{\phi}} \sqrt{-\operatorname{det} \hat{g}}+\int_{M_{10}} \hat{B}^{(10)} .
$$

In this case, using eqs. (5.3), one obtains that the upper sign choice in eq. (5.1) leads to an action with no goldstino, while the lower sign choice leads to a VA action, and in both 
cases no constraint is required. This means that the untruncated action is $\kappa$-symmetric, and thus we argue that this is the correct action for an NS9-brane. More precisely, the complete action would result from rescaling the S-dual of the lagrangian of eq. (2.7), and in order to compute this, one should know how $C^{(8)}$ and $C^{(6)}$ transform under S-duality. This analysis is currently under investigation, and we expect that it would shed some light on the problem of studying the S-dual of a D7-brane as well. Anyway, we do not expect the results of this section to be altered by the inclusion of additional terms, since we do not see how the constraint of eq. (4.17) can be removed modifying $B^{(10)}$ by the inclusion of other bulk fields.

One could also discuss the S-dual of this picture, starting from the action of eq. (5.6), and then performing an S-duality transformation. The result is that one ends up with the action

$$
S=-\int_{M_{10}} d^{10} \xi e^{-3 \hat{\phi}} \sqrt{-\operatorname{det} \hat{g}}+\int_{M_{10}} e^{-2 \hat{\phi}} \hat{C}^{(10)}
$$

Again, $\kappa$-symmetry corresponds to the existence of a $Z_{2}$ truncation removing the goldstino completely, and one can show that this happens only after imposing the constraint for $C^{(10)}$ in eq. (4.17). The picture is thus completely symmetric, since from this low-energy point of view assuming that the D9-brane action is (2.7) instead of (5.7) is S-dual to assuming that the action for an NS9-brane is (5.6) instead of (5.2).

At the end of this section, we want to determine the supersymmetric action for an NS9-brane in type-IIA, where again $\kappa$-symmetry corresponds to the vanishing of all the goldstino terms in the suitably $Z_{2}$-truncated action. The 10 -dimensional $Z_{2}$-truncation projects out all the RR fields, acting on the fermions as

$$
\begin{aligned}
& \psi_{\mu}= \pm \Gamma_{11} \psi_{\mu} \\
& \lambda=\mp \Gamma_{11} \lambda .
\end{aligned}
$$

From the supersymmetry transformation of eq. (3.4) we obtain

$$
\begin{aligned}
\hat{B}_{\mu_{1} \ldots \mu_{10}}^{(10)} & =B_{\mu_{1} \ldots \mu_{10}}^{(10)}-10 e^{-2 \phi} \bar{\theta} \Gamma_{\left[\mu_{1} \ldots \mu_{9}\right.} \psi_{\left.\mu_{10}\right]}+e^{-2 \phi} \bar{\theta} \Gamma_{\mu_{1} \ldots \mu_{10}} \lambda \\
& -5 e^{-2 \phi} \bar{\theta} \Gamma_{\left[\mu_{1} \ldots \mu_{9}\right.} D_{\left.\mu_{10}\right]} \theta+\ldots
\end{aligned}
$$

The truncation acts on the world-volume fields as usual: the vector $V^{i}$ is projected out, while $\theta$ transforms in the same way as $\psi$. The final result is that the truncated action

$$
S=-\int_{M_{10}} d^{10} \xi e^{-2 \hat{\phi}} \sqrt{-\operatorname{det} \hat{g}}+\int_{M_{10}} \hat{B}^{(10)}
$$


is supersymmetric choosing the upper sign in eq. (5.8), while supersymmetry is spontaneously broken if one chooses the lower sign. It can be shown that, after an $S^{1}$ reduction, T-duality relates this action with the one of eq. (5.6).

\section{Conclusions}

The starting point of this paper was a continuation of [24], where the results of [23] were generalized to a curved background, showing that the possible type-I truncations of typeIIB are in correspondence with the possible consistent type-I strings in $D=10$. We showed here that the same results apply to the $\mathrm{D}=9$ truncations of type-IIA, in accordance with T-duality. We then proceeded constructing the $\kappa$-symmetric spacetime-filling branes that are charged with respect to the NS 10-forms of type-IIB and type-IIA.

In [29] it was argued that S-duality of type-IIB implies the existence of NS9-branes, that together with the D9-branes form an $S L(2, Z)$ doublet. From the standard Weylrescaling argument, it turns out that the tension of these branes appears to scale like $1 / g_{S}^{4}$ in the string frame. Here we have argued that the actual tension of these branes scales like $1 / g_{S}^{2}$, like the other solitonic NS objects, namely NS5-branes. The solution of the paradox is that the doublet of NS and RR 10-form potentials does not transform covariantly under $S L(2, Z)$. It is therefore not possible to derive the action for an NS9-brane performing a Weyl rescaling. In $[29,30]$ it was also conjectured that S-duality implies the existence of a dual of the orientifold projection, and the $S O(32)$ heterotic theory should result from this projection, after the introduction of 32 NS9-branes. This projection would naturally act like $\sigma_{3}$ on the fermion doublets, since in the heterotic theory the fermions come only from the left sector. We do not expect this truncation to be an ordinary $Z_{2}$ orbifold, since it is well know that a $Z_{2}$ orbifold of type-IIB gives rise to type-IIA. In any case, if there is a way of deriving the heterotic $S O(32)$ theory from type-IIB, we expect that the 'twisted' sector of the projection would correspond to inserting $\kappa$-symmetric branes, that would therefore have the structure of eq. (5.6). In any case, should a brane interpretation of the heterotic theory be possible, a natural question would arise, namely what is the heterotic string equivalent of brane supersymmetry breaking ${ }^{10}$.

Similar arguments hold for the IIA case. Since the type-IIA superstring and the $E_{8} \times E_{8}$ heterotic theory have both an M-theory origin [40, 41], it has also been conjectured [29]

\footnotetext{
${ }^{10} \mathrm{I}$ am grateful to E. Dudas for discussions about this point.
} 
that the $E_{8} \times E_{8}$ heterotic theory can arise in ten dimensions from a projection of type-IIA, that would result in the low-energy action in a $Z_{2}$ truncation removing the RR fields. We emphasize again that if this projection exists, it cannot act as a $Z_{2}$ orbifold of type-IIA, since such an orbifold gives rise to type-IIB. The 'twisted' sector of the heterotic theory would result in this case form the insertion of NS9-branes. Starting from type-IIB and using Weyl-rescaling arguments, it has been argued that T-duality would imply that the tension of this branes, apart from having an $e^{-4 \phi}$ dilaton dependance, is proportional to $R^{3}$, where $R$ is the radius of the isometry direction [31], and this would mean that they are not defined in 10 uncompactified dimensions. The NS9-brane, as well as the D8-brane, would then result from a 9-brane in M-theory whose effective action and target space solution [42] can be written only if the 11-dimensional supergravity has an isometry, and thus cannot be covariant in 11-dimensions. Again, the $\kappa$-symmetric spacetime-filling brane we obtained in this paper has instead an $e^{-2 \phi}$ dilaton dependance, and it is related by T-duality to the type-IIB NS-brane of eq. (5.6). This different scaling with respect to the one of [31] implies that this NS9-brane and the D8-brane can not have a common M-theory origin. Since the field-strength of a 10-form in 11 dimensions would be dual to a cosmological constant, this result is basically rephrasing the fact that no cosmological constant can be included in 11-dimensional supergravity [43], and Romans IIA supergravity can not be obtained by dimensional reduction from 11 dimensions. After compactification on a 2-torus M-theory is related to type-IIB by T-duality, and thus this picture is the T-dual analogous of the type-IIB picture, where S-duality is broken by the presence of spacetime-filling branes.

Finally, it would be interesting to see if the S-duality rules of this paper can be used to understand the strong coupling behavior of the D7-branes. In [44] it was shown that D7-branes of type-IIB belong to a triplet of 7-branes. One could then determine a supersymmetric effective action for these branes requiring $\kappa$-symmetry, and relate them to the half-BPS 7-brane solutions of type-IIB supergravity [45, 46, 47]. This analysis is currently under investigation. 


\section{Acknowledgments}

I am grateful to E. Bergshoeff, M. Bianchi, E. Dudas, M. Green and G. Pradisi for discussions. This work is supported by a European Commission Marie Curie Postdoctoral Fellowship, Contract MEIF-CT-2003-500308.

\section{References}

[1] J. Polchinski, "Dirichlet-Branes and Ramond-Ramond Charges," Phys. Rev. Lett. 75 (1995) 4724 [arXiv:hep-th/9510017].

[2] J. Dai, R. G. Leigh and J. Polchinski, "New Connections Between String Theories," Mod. Phys. Lett. A 4 (1989) 2073.

[3] E. S. Fradkin and A. A. Tseytlin, "Nonlinear Electrodynamics From Quantized Strings," Phys. Lett. B 163 (1985) 123.

[4] A. Abouelsaood, C. G. Callan, C. R. Nappi and S. A. Yost, "Open Strings In Background Gauge Fields," Nucl. Phys. B 280 (1987) 599.

[5] M. Cederwall, A. von Gussich, B. E. Nilsson and A. Westerberg, "The Dirichlet superthree-brane in ten-dimensional type IIB supergravity," Nucl. Phys. B 490 (1997) 163 [arXiv:hep-th/9610148].

[6] M. Aganagic, C. Popescu and J. H. Schwarz, "D-brane actions with local kappa symmetry," Phys. Lett. B 393 (1997) 311 [arXiv:hep-th/9610249].

[7] M. Cederwall, A. von Gussich, B. E. Nilsson, P. Sundell and A. Westerberg, "The Dirichlet super-p-branes in ten-dimensional type IIA and IIB supergravity," Nucl. Phys. B 490 (1997) 179 [arXiv:hep-th/9611159].

[8] E. Bergshoeff and P. K. Townsend, "Super D-branes," Nucl. Phys. B 490 (1997) 145 [arXiv:hep-th/9611173].

[9] M. Aganagic, C. Popescu and J. H. Schwarz, "Gauge-invariant and gauge-fixed Dbrane actions," Nucl. Phys. B 495 (1997) 99 [arXiv:hep-th/9612080]. 
[10] W. Siegel, "Hidden Local Supersymmetry In The Supersymmetric Particle Action," Phys. Lett. B 128 (1983) 397.

[11] M. B. Green and J. H. Schwarz, "Covariant Description Of Superstrings," Phys. Lett. B 136 (1984) 367.

[12] D. V. Volkov and V. P. Akulov, "Is The Neutrino A Goldstone Particle?," Phys. Lett. B 46 (1973) 109; U. Lindstrom and M. Rocek, "Constrained Local Superfields," Phys. Rev. D 19 (1979) 2300; S. Samuel and J. Wess, "A Superfield Formulation Of The Nonlinear Realization Of Supersymmetry And Its Coupling To Supergravity," Nucl. Phys. B 221 (1983) 153, "Realistic Model Building With The Akulov-Volkov Superfield And Supergravity," Nucl. Phys. B 226 (1983) 289, "Secret Supersymmetry," Nucl. Phys. B 233 (1984) 488; J. Bagger and A. Galperin, "Matter couplings in partially broken extended supersymmetry," Phys. Lett. B 336 (1994) 25 [hep-th/9406217].

[13] A. Sagnotti, in Cargese '87, "Non-Perturbative Quantum Field Theory", eds. G.Mack et al (Pergamon Press, 1988), p. 521, "Open Strings And Their Symmetry Groups," arXiv:hep-th/0208020; G. Pradisi and A. Sagnotti, "Open String Orbifolds," Phys. Lett. B 216 (1989) 59; M. Bianchi and A. Sagnotti, "On The Systematics Of Open String Theories," Phys. Lett. B 247 (1990) 517; "Twist symmetry and open string Wilson lines," Nucl. Phys. B 361 (1991) 519; M. Bianchi, G. Pradisi and A. Sagnotti, "Toroidal compactification and symmetry breaking in open string theories," Nucl. Phys. B 376 (1992) 365.

[14] M. B. Green and J. H. Schwarz, "Anomaly Cancellation In Supersymmetric D=10 Gauge Theory And Superstring Theory," Phys. Lett. B 149 (1984) 117; "Infinity Cancellations In SO(32) Superstring Theory," Phys. Lett. B 151 (1985) 21.

[15] N. Ohta, "Cancellation Of Dilaton Tadpoles And Two Loop Finiteness In SO(32) Type I Superstring," Phys. Rev. Lett. 59 (1987) 176.

[16] J. Polchinski and Y. Cai, "Consistency Of Open Superstring Theories," Nucl. Phys. B 296 (1988) 91.

[17] S. Sugimoto, "Anomaly cancellations in type I D9-D9-bar system and the USp(32) string theory," Prog. Theor. Phys. 102 (1999) 685 [arXiv:hep-th/9905159].

[18] M. Bianchi and J. F. Morales, "Anomalies and tadpoles," JHEP 0003 (2000) 030 [arXiv:hep-th/0002149]. 
[19] I. Antoniadis, E. Dudas and A. Sagnotti, "Brane supersymmetry breaking," Phys. Lett. B 464 (1999) 38 [arXiv:hep-th/9908023].

[20] E. Dudas and J. Mourad, "Consistent gravitino couplings in non-supersymmetric strings," Phys. Lett. B 514 (2001) 173 [arXiv:hep-th/0012071].

[21] W. Fischler and L. Susskind, "Dilaton Tadpoles, String Condensates And Scale Invariance," Phys. Lett. B 171 (1986) 383; "Dilaton Tadpoles, String Condensates And Scale Invariance. 2," Phys. Lett. B 173 (1986) 262.

[22] E. Dudas, G. Pradisi, M. Nicolosi and A. Sagnotti, "On tadpoles and vacuum redefinitions in string theory," arXiv:hep-th/0410101.

[23] E. Bergshoeff, M. de Roo, B. Janssen and T. Ortin, "The super D9-brane and its truncations," Nucl. Phys. B 550 (1999) 289 [arXiv:hep-th/9901055].

[24] F. Riccioni, "Truncations of the D9-brane action and type-I strings," Phys. Lett. B 560 (2003) 223 [arXiv:hep-th/0301021].

[25] G. Pradisi and F. Riccioni, "Geometric couplings and brane supersymmetry breaking," Nucl. Phys. B 615 (2001) 33 [arXiv:hep-th/0107090].

[26] E. Bergshoeff, R. Kallosh, T. Ortin, D. Roest and A. Van Proeyen, "New formulations of D = 10 supersymmetry and D8 - O8 domain walls," Class. Quant. Grav. 18 (2001) 3359 [arXiv:hep-th/0103233].

[27] C. M. Hull and P. K. Townsend, "Unity of superstring dualities," Nucl. Phys. B 438 (1995) 109 [arXiv:hep-th/9410167].

[28] J. Polchinski and E. Witten, "Evidence for Heterotic - Type I String Duality," Nucl. Phys. B 460 (1996) 525 [arXiv:hep-th/9510169].

[29] C. M. Hull, "Gravitational duality, branes and charges," Nucl. Phys. B 509 (1998) 216 [arXiv:hep-th/9705162].

[30] C. M. Hull, "The non-perturbative SO(32) heterotic string," Phys. Lett. B 462 (1999) 271 [arXiv:hep-th/9812210].

[31] E. Bergshoeff, E. Eyras, R. Halbersma, J. P. van der Schaar, C. M. Hull and Y. Lozano, "Spacetime-filling branes and strings with sixteen supercharges," Nucl. Phys. B 564 (2000) 29 [arXiv:hep-th/9812224]. 
[32] I. A. Bandos, J. A. de Azcarraga, J. M. Izquierdo and J. Lukierski, "An action for supergravity interacting with super-p-brane sources," Phys. Rev. D 65 (2002) 021901 [arXiv:hep-th/0104209]; " $\mathrm{D}=4$ supergravity dynamically coupled to a massless superparticle in a superfield Lagrangian approach," arXiv:hep-th/0207139; "On dynamical supergravity interacting with super-p-brane sources," arXiv:hep-th/0211065.

[33] E. A. Bergshoeff, M. de Roo and A. Sevrin, "Non-Abelian Born-Infeld and kappasymmetry," J. Math. Phys. 42 (2001) 2872 [arXiv:hep-th/0011018].

[34] J. Gomis, K. Kamimura and P. K. Townsend, "Non-relativistic superbranes," arXiv:hep-th/0409219.

[35] L. J. Romans, "Massive N=2a Supergravity In Ten-Dimensions," Phys. Lett. B 169 (1986) 374 .

[36] E. Bergshoeff and M. De Roo, "D-branes and T-duality," Phys. Lett. B 380 (1996) 265 [arXiv:hep-th/9603123].

[37] M. B. Green, C. M. Hull and P. K. Townsend, "D-Brane Wess-Zumino Actions, TDuality and the Cosmological Constant," Phys. Lett. B 382 (1996) 65 [arXiv:hepth/9604119].

[38] E. Bergshoeff, C. M. Hull and T. Ortin, "Duality in the type II superstring effective action," Nucl. Phys. B 451 (1995) 547 [arXiv:hep-th/9504081].

[39] J. H. Schwarz, "Covariant Field Equations Of Chiral N=2 D = 10 Supergravity," Nucl. Phys. B 226 (1983) 269.

[40] E. Witten, "String theory dynamics in various dimensions," Nucl. Phys. B 443 (1995) 85 [arXiv:hep-th/9503124].

[41] P. Horava and E. Witten, "Heterotic and type I string dynamics from eleven dimensions," Nucl. Phys. B 460 (1996) 506 [arXiv:hep-th/9510209].

[42] E. Bergshoeff and J. P. van der Schaar, "On M-9-branes," Class. Quant. Grav. 16 (1999) 23 [arXiv:hep-th/9806069].

[43] K. Bautier, S. Deser, M. Henneaux and D. Seminara, "No cosmological D = 11 supergravity," Phys. Lett. B 406 (1997) 49 [arXiv:hep-th/9704131].

[44] P. Meessen and T. Ortin, " $\mathrm{An} \operatorname{Sl}(2, \mathrm{Z})$ multiplet of nine-dimensional type II supergravity theories," Nucl. Phys. B 541 (1999) 195 [arXiv:hep-th/9806120]. 
[45] G. W. Gibbons, M. B. Green and M. J. Perry, "Instantons and Seven-Branes in Type IIB Superstring Theory," Phys. Lett. B 370 (1996) 37 [arXiv:hep-th/9511080].

[46] M. B. Einhorn and L. A. Pando Zayas, "On seven-brane and instanton solutions of type IIB," Nucl. Phys. B 582 (2000) 216 [arXiv:hep-th/0003072].

[47] E. Bergshoeff, U. Gran and D. Roest, "Type IIB seven-brane solutions from nine-dimensional domain walls," Class. Quant. Grav. 19 (2002) 4207 [arXiv:hepth/0203202]. 